\title{
CULTURA POLÍTICA Y PARLAMENTO EN EL PERÚ (2011-2016). LA CORRUPCIÓN EN LA PERCEPCIÓN DE LOS ESTUDIANTES UNIVERSITARIOS DE LIMA ${ }^{1}$
}

\author{
Omar YALLE QUINCHO \\ Universidad Ricardo Palma \\ omar.yalle@urp.edu.pe
}

\section{RESUMEN}

El objeto de la presente investigación es conocer la cultura política sobre el Parlamento en el Perú (2011-2016), a partir de la percepción de los estudiantes universitarios de cuatro universidades de Lima. Se plantea explorar a manera de estudio piloto, sobre las ideas que tienen respecto a la representación parlamentaria y los problemas éticos y de corrupción de algunos representantes, así como los imaginarios que construyen en torno a ello.

\section{PALABRAS CLAVES}

Cultura Política. Parlamento. Corrupción. Organización Política

\section{POLITICAL CULTURE AND PARLIAMENT IN PERU (2011-2016). CORRUPTION IN THE PERCEPTION OF UNIVERSITY STUDENTS IN LIMA}

\section{ABSTRACT}

The purpose of this work is to know the political culture of Parliament in Peru (20112016), based on the perception of university students from four universities in Lima. It is proposed to explore, as a pilot study, the ideas they have regarding parliamentary representation and the ethical and corruption problems of some representatives, as well as the imaginaries that they build around it.

\section{KEYWORDS}

Political culture. Parliament. Corruption. Political organization

Recibido: $20 / 04 / 2020$

Aprobado:29/06/2020

1 El presente trabajo es una versión corregida del borrador del informe de investigación titulado: Cultura, Política y Parlamento en el Perú (2011-2016). La Corrupción en la Percepción de los Estudiantes Universitarios de Lima, presentado al Centro de Investigación de la Universidad Ricardo Palma, como parte del Programa Anual de Investigación 2016. Agradezco el apoyo del Vicerrectorado de Investigación en la persona del Dr. Hugo Sánchez, al Dr. José Martínez, entonces director del Centro de Investigación y al Dr. Pedro Jacinto Pazos, Profesor investigador de la Universidad. Así mismo, a la Dra. María Ulfe, por su asesoramiento en esta temática de investigación, en la Pontificia Universidad Católica del Perú. 


\section{INTRODUCCIÓN}

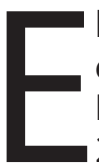

I Parlamento es un poder del Estado en una democracia constitucional, encargado de dar, interpretar, modificar y derogar leyes (Constitución Política del Perú 1993, art. 102, Reglamento del Congreso de la República 2020). Tiene 4 funciones: la función normativa, de gobierno interno, de control y de representación. La función normativa es la dación de leyes, donde la constitución es la de más alta jerarquía. La función de gobierno interno se da cuando el legislativo decide de manera autónoma, sin ninguna intromisión de otro órgano. Es un gobierno interno dentro del equilibrio de poderes. La función de control o de fiscalización que realiza, principalmente, del órgano ejecutivo del Estado o de otros poderes e instituciones, como parte del poder constituido. Así, examina la corrección jurídica y política de otros órganos del Estado. Y de representación. Es la sociedad peruana el que está legislando en el parlamento. Lo hace mediante el congreso a través de sus representantes (Delgado-Guembes 2012; Valencia 2007)

El parlamento también es el escenario de la ritualización representativa, un espacio de los valores y creencias, los discursos y las acciones, que se generan en la relación representante/representado. Es también un espacio mediatizado de la función pública. Su sentido emana del poder constituyente, es decir, la sociedad le otorga legitimidad a través del voto ciudadano para las responsabilidades políticas del poder estatal.

Los estudios tratados sobre el parlamento desde distintos ejes temáticos han privilegiado una mirada etnocéntrica, a partir de una construcción teórico empírico, del funcionamiento y la representación legislativa (Bernales, 1990 y 2002; DEA/UARM 2009, Campos 2017, Casado 2018, Gumaray y Prado 2020), así como desde otras miradas desde las ciencias sociales (Ugarteche 2006, Portocarrero 2010, Mujica 2013, Mujica \& Zevallos 2016) De otro modo, la corrupción y su relación con el parlamento, se ha relacionado con el campo temático de la eficacia y gobernabilidad, la democracia y los sistemas de representación en el Parlamento. (Meléndez 2011, Proética 2015, Huber 2017).

\section{a. Ideas sobre la noción de corrupción política}

En un trabajo anterior (Yalle 2019), señalamos que la corrupción constituye un problema estructural, complejo y diferenciado. Las definiciones asignadas, apelan a diferentes parámetros como: la moral, el derecho, la percepción de la ciudadanía, la racionalidad económica y las normas e instituciones comprendidas culturalmente, otorgandole una definición semiótica, es decir, que se presenta desde distintas formas de entenderla (Morelos 2015).

Esta se manifiesta como una actitud diferenciada entre las personas, desde una ubicación jerárquica, en una relación de poder, que busca el beneficio de uno de ellos o de ambos, comprendiendo acciones de deslealtad frente al interés natural que ocupan en el grupo social (Calsamiglia, 2015; 19). Como señala Laporta (1997), dentro de esta relación los elementos que conforman parte del mismo son: los costos de corrupción: el corruptor, el corrupto, el beneficio, la 
posible expectativa de una contraprestación y el secreto. Sostiene además, que "...consiste en la violación limitada de una obligación por parte de uno o más decisores con el objeto de obtener un beneficio personal extra posicional...". (p. $52)$.

En su esfera no privada, implica el abuso del poder, que se concentra en una persona o grupos de personas, bajo la misma lógica que el ámbito público. Esto se da cuando se asume una función pública. Pero también puede verse recreado en una relación privada donde por acuerdo o por un sentido de dependencia personal o de desventaja, puede establecerse un beneficio individual o mutuo(Klitgaard 1990). Es una relación de tipo contractual, donde no operan los valores desde una acción positiva. Así, la institucionalidad política, desde los actos individuales, tiende a pervertir la fragilidad de lo público, como objeto del bien común y que es el Estado representado en sus instituciones, convirtiéndolo en cleptocrático y generando una relación entre la sociedad política y la sociedad civil, con un sentido de anomia.

La corrupción es una condicionante de las actitudes positivas de las personas como parte del ejercicio en la función pública. Se ha entendido generalmente desde la esfera "oficial", difiriendo de una apreciación sociocultural, como un hecho social total. Es decir, como debe entenderse la influencia de las estructuras de la sociedad que se ejercen en los pensamientos y acciones de los individuos, jerarquizándolo, y donde cada uno cumple un rol determinado según las normas y valores del grupo en el que se desenvuelven. De tal modo, que podemos hablar del hecho social como externo y coercitivo. La manera como entienden la corrupción los ciudadanos, por ejemplo, desde las practica cotidianas, donde el concepto mismo puede verse como polisémico y trascendiendo lo económico y jurídica-normativo (Durkheim 1988; Ugarteche 2006; Huber 2008).

Su análisis constituye de por sí una variable compleja, debido a su inmensa carga ideológica y valorativa. En algunos casos la vaguedad, la ambigüedad, el carácter emotivo del término, son obstáculos que le permiten delimitar mejor el concepto. Uno de estos aspectos está relacionada con la función pública. El funcionario o burócrata se ve en ventaja de hacer el mal uso de su autoridad en beneficio personal. Maximiza sus beneficios y concibe su puesto como un negocio. (Calsamiglia 2015, 27).

En ese sentido, el sujeto participa y privilegia la acción vedada de los distintos actores públicos y privados, subordinando y deslegitimando las acciones personales en las relaciones humanas y sociales. Subordinación y deslegitimación de la acción social que tiene un costo-beneficio, convirtiendo el mismo en un acto amoral y delictivo. Por lo tanto, el problema de la corrupción no solo tiene un sentido semiológico univoco, por el contrario, se presenta como una recreación vital de la racionalidad de los patrones de comportamiento en los grupos sociales, que permiten la dinámica de las instituciones políticas como el parlamento (Mujica 2016). Son estrategias de discurso y acciones elaboradas con sentido integrador de relaciones de poder. 


\section{b. La representación parlamentaria}

La representación parlamentaria a través del tiempo, ha tenido un rol performativo. En la década de los noventa, se hacen manifiesta nuevas formas de pensar y hacer política. Se sientan las bases para el surgimiento de nuevas formas de representación, explicados por el colapso de los llamados viejos partidos ideológicos, el despliegue de un individualismo pragmático, el cinismo como cualidad política, el auge de los denominados movimientos independientes, la mercantilización de la política, el trasfondo jurídico de su legalidad y su cuestionada legitimidad.

La crisis de representación parlamentaria se explica entonces, por la crisis de los partidos políticos y de las ideologías, el individualismo pragmático ante una realidad movediza y de perspectivas más individuales que colectivas. Como consecuencia de este pragmatismo, la sustentación del cinismo, la mentira, la banalización, la venalidad, monopolizan la capacidad dinámica de la corrupción. Hoy podemos observar como estas nuevas formas de pensar y hacer política, se individualizan en casos más de tipo personal, ligados al comportamiento antiético y la conducta delictiva como parte de la función representativa.

En el Parlamento peruano, para el periodo 2011-2016, se eligieron a 130 congresistas, distribuidos inicialmente en seis agrupaciones políticas, como son: Gana Perú 47, Fuerza 2011 37, Perú Posible 21, Solidaridad Nacional 9, Alianza Por el Gran Cambio 13 y el Partido Aprista Peruano (JNE 2011). De los cientotreinta congresistas, hasta antes de cumplir los 100 días de gestión, 11 ya tenían iniciado procesos judiciales, en algunos casos faltas y delitos, cometidos antes de la función legislativa. A noviembre de 2012 ya se contabilizaba en 46 los parlamentarios con algún tipo de acusación ética y dolosa.

Desde esa perspectiva, el presente estudio se centra en el parlamento desde la percepción de los universitarios, para el periodo 2011-2016. Al respecto nos planteamos las siguientes preguntas:

1. Como parte de la cultura política de la ciudadanía ¿qué percepciones tienen los universitarios sobre el parlamento peruano?

2. ¿Qué casos conocidos permiten comprender el tema de la corrupción como una práctica de la actividad parlamentaria en el periodo 2011-2016?

3. ¿Qué imaginarios y discursos, construyen sobre la corrupción parlamentaria, los estudiantes universitarios de Lima?

\section{MATERIAL Y MÉTODO}

El presente trabajo se sustenta en un cuestionario de encuesta codificado, con el fin de obtener información sobre una parte de la población (universitarios). La información recogida se emplea en el análisis cuantitativo, con el fin de identificar 
y conocer el problema planteado, de forma parcial. Es aplicado a estudiantes de cuatro universidades limeñas: tres privadas y una pública: la Pontificia Universidad Católica del Perú, la Universidad Ricardo Palma, la Universidad Peruana Los Andes Filia-Lima, y la Universidad Nacional Mayor de San Marcos.

En total seaplicaron 250 encuestas, mediante un diseño muestral, con la modalidad al azar simple, y a criterio del investigador, realizado a varones y mujeres entre 18 y 45 años, distribuidas de la siguiente manera: 45 en la PUCP, 60 en la URP, 60 en la UPLA Filial-Lima, y 85 en la UNMSM. El cuestionario consto de trece preguntas con alternativas y tres preguntas con opción de respuesta múltiple. Para el presente trabajo por motivo de requerimiento para su publicación, presentamos solo 8 cuadros.

\section{Tipo de investigación}

Por el tipo de investigación, el presente estudio reúne las condiciones metodológicas de una investigación básica.

\section{Nivel de investigación}

De acuerdo con la naturaleza del nivel de investigación, reúne las características de un estudio exploratorio y descriptivo.

\section{Método de la investigación}

Mixto. Se basará en la codificación de datos a partir del uso de encuestas aplicadas para la clasificación e interpretación.

\section{Diseño de la investigación.}

Descriptivo.

\section{Técnicas:}

- Sistematización bibliográfica y hemerográfica.

- Encuestas.

\section{Instrumentos:}

- Guía de encuestas.

\section{RESULTADOS}

En esta sección del trabajo presentamos 8 cuadros que nos permitieron responder a las preguntas planteadas como parte del problema de investigación. Las barras de los cuadros presentan los resultados en porcentajes de nuestra encuesta. 


\section{Cuadro $\mathrm{N}^{\circ} 1$}

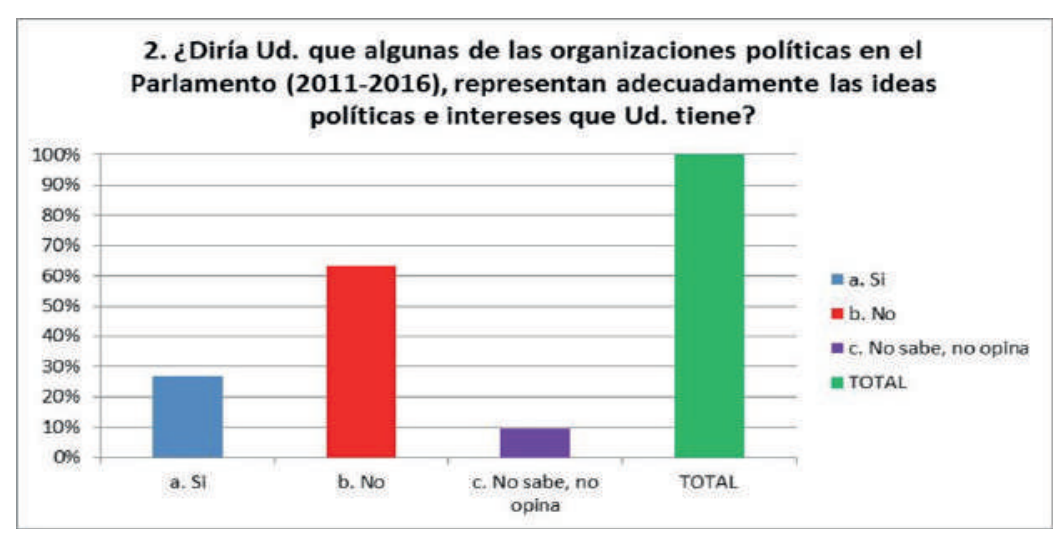

En el Cuadro $\mathrm{N}^{\circ} 1$, se observa los porcentajes respecto a la percepción de las organizaciones y representación política, respecto a los intereses que los estudiantes universitarios tienen. Estas no representan sus ideas e interés mayoritariamente $(63.3 \%)$, frente a un $(27 \%)$ que considera que los partidos políticos en el parlamento representan sus ideas políticas e intereses. Existe un $(9.6 \%)$ que se mantiene al margen de estas dos expectativas.

\section{Cuadro $\mathrm{N}^{\circ} 2$}

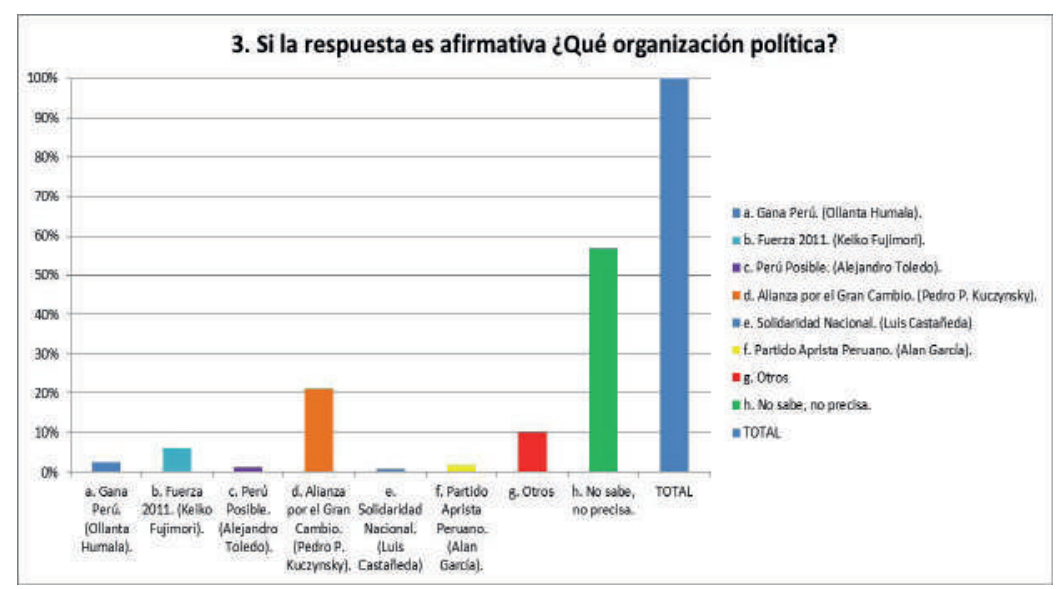

El estudiante universitario de Lima, como se infiere del cuadro anterior, en su mayoría no se ve identificado por las organizaciones políticas al parlamento 2011-2016. Dado que la encuesta se realizó el año 2016, se justifica. Así, el 21\% opto entre las preferencias, por la organización política Alianza por el Gran Cambio. En segundo lugar, encontramos a Fuerza 2011 con un (6\%). Algo más distante, nos muestran los resultados de la encuesta a Gana Perú con (2.4\%), al Partido Aprista Peruano (1.6\%), Perú Posible (1.2\%) y Solidaridad Nacional con (0.8\%). Sin embargo, un sector considerable de encuestados considera otras organizaciones políticas sin especificar $(10.1 \%)$, y un importante (57\%), no sabe y no precisa. 


\section{Cuadro $\mathrm{N}^{\circ} 3$}

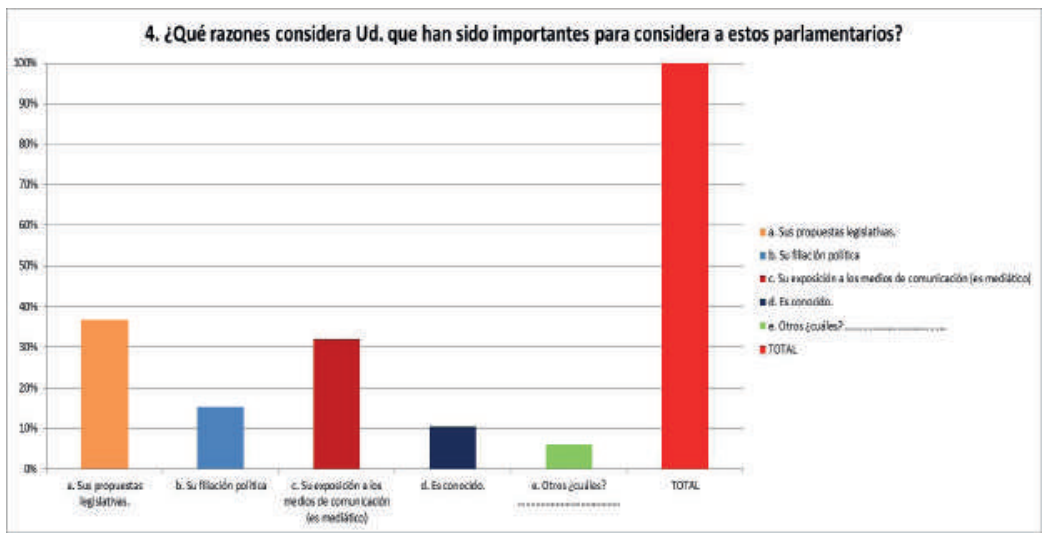

En el cuadro $\mathrm{N}^{\circ} 3$, se observa los porcentajes sobre las razones por la que los estudiantes universitarios consideran importante al momento de elegir a sus representantes. Están en primer lugar, que estos tengan propuestas legislativas (36.6\%). Un (31.8\%), considera que la exposición a los medios de comunicación como la televisión de señal abierta, son los más proclives a ser elegidos. Un (15\%) considera importante la filiación política partidaria. Así mismo, un (10\%) cree que el candidato al parlamento es una persona conocida, y un (5.9\%), considera otras las razones de elección de sus representantes.

\section{Cuadro $\mathrm{N}^{\circ} 4$}

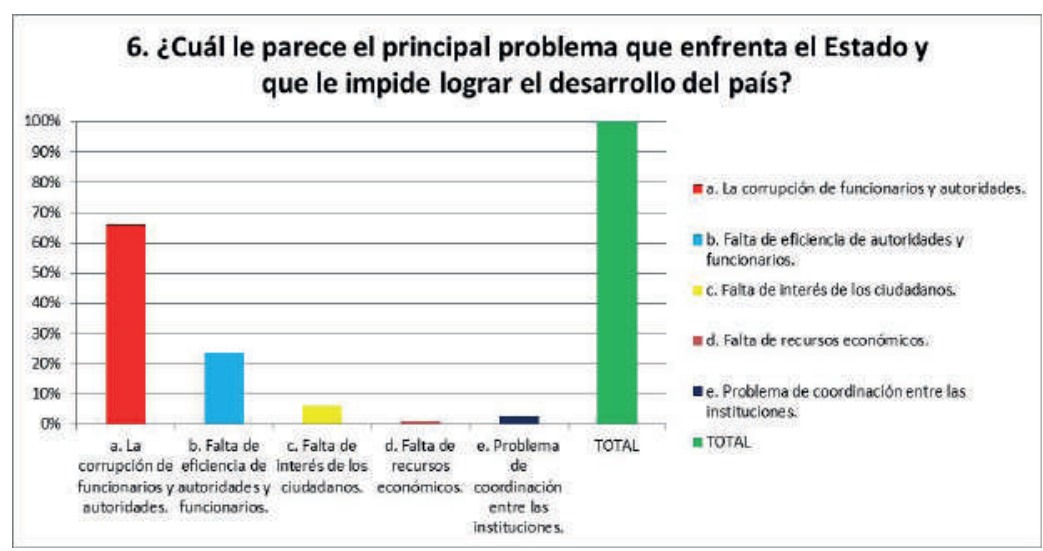

La corrupción constituye el principal problema que enfrenta el Estado y que le impide lograr su desarrollo como país (67\%). Esto se complementa con la falta de eficiencia de autoridades y funcionarios (23\%). La falta de interés de los ciudadanos (6\%) constituye otro factor por el cual, las acciones de los agentes del Estado no son fiscalizadas. Un sector de estudiantes universitarios encuestados cree que es la falta de recursos (1.2\%), desde del Estado, como dotar de un mayor presupuesto a los gobiernos locales y regionales, lo que permitiría un mejor funcionamiento del Estado. El (2.8\%) señala que el problema que enfrena el Estado y no permite logra su 
desarrollo es la falta de coordinación entre las instituciones.

\section{Cuadro $\mathrm{N}^{\circ} 5$}

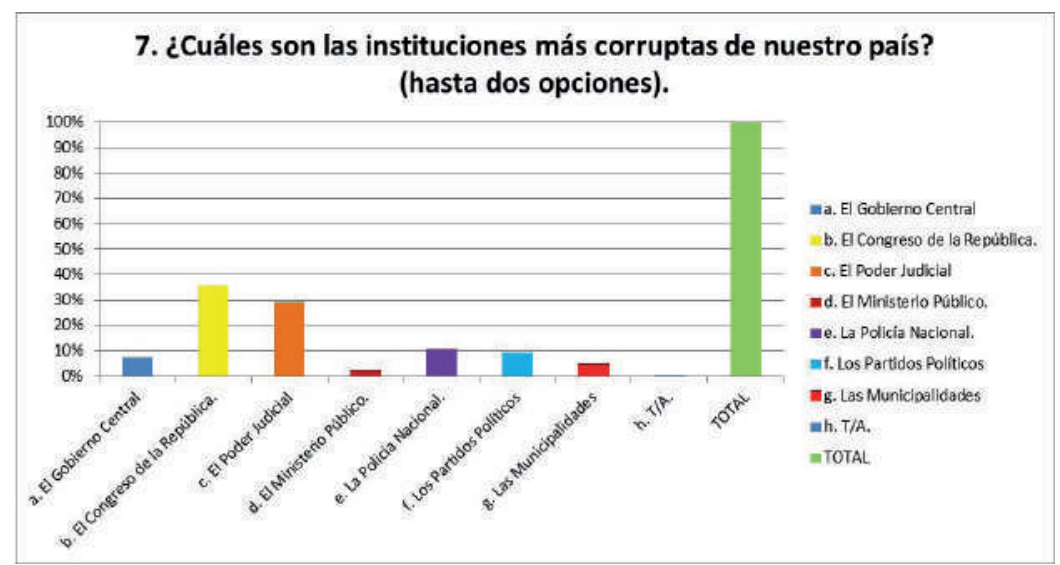

En el cuadro $\mathrm{N}^{\circ} 5$, se presentan los porcentajes sobre ¿cuáles son las instituciones más corruptas en nuestro país? Dentro de la percepción de los estudiantes universitarios, se encuentra en primer lugar, el Congreso de la República (35.7\%). En segundo lugar, el Poder Judicial (29.2\%). En tercer lugar, ubicamos a la Policía Nacional con un (10.5\%). El (9.2\%) percibe también que la corrupción se manifiesta en los partidos políticos. Un (7.3\%) considera que esta se da desde las esferas del Poder Ejecutivo. Siguen las municipalidades con un (4.7\%).

El Ministerio Público es otra de las instituciones consideradas también como corruptas en el país con un (2.6\%). Así mismo, un (0.5\%), considera que todas las instituciones anteriores se caracterizan por tener esta naturaleza.

\section{Cuadro $\mathrm{N}^{\circ} 6$}

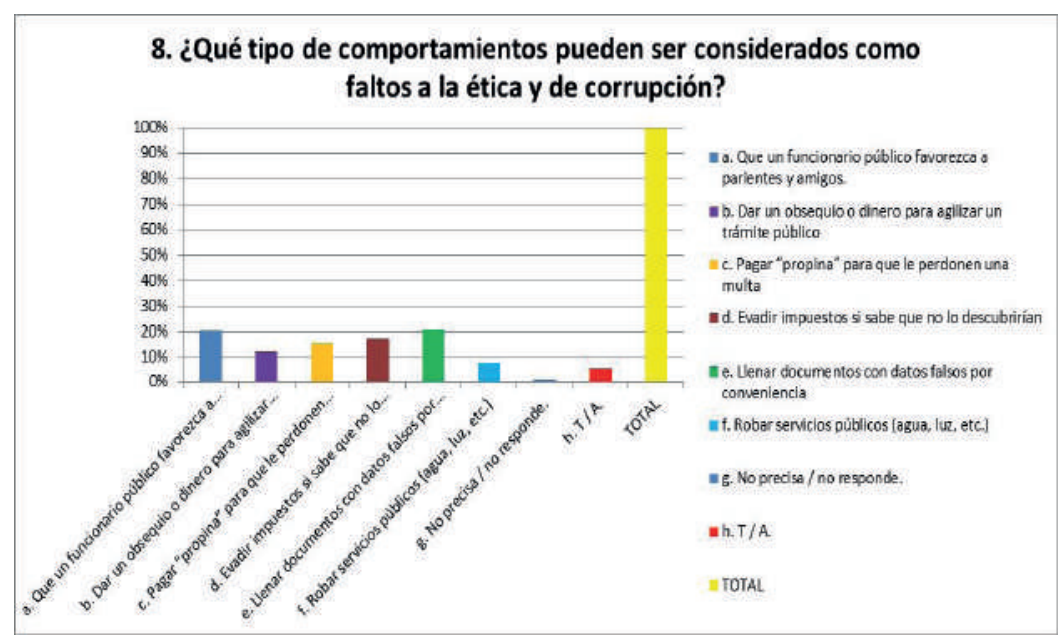


En el cuadro $N^{\circ} 6$, respecto al tipo de comportamiento que pueden ser considerados como faltos a la ética y de corrupción, tenemos que un (20.3\%) de los encuestados, considera que un funcionario público favorezca a parientes y amigos. Un (17.1\%) considera que evadir impuesto si se sabe que no lo descubrirán, constituye otro de los comportamientos faltos a la ética y vistos como corrupto. El (15.5\%), señala que pagar propina para que le perdonen la multa es también un acto de corrupción. Un (11.9\%) considera que dar un obsequio o dinero para agilizar un trámite público también lo es.

Un (7.5\%), considera que robar servicios públicos como el agua, la luz o el cable, son actos contra la ética y considerados como corruptos. Esto se entiende como el acuerdo entre el beneficiario y el dador o facilitador del servicio, sea este un funcionario de la institución pública o privada. El (1.1\%) no sabe y no precisa. Y el (5.5\%) considera, todos los comportamientos anteriormente mencionados.

\section{Cuadro $\mathrm{N}^{\circ} 7$}

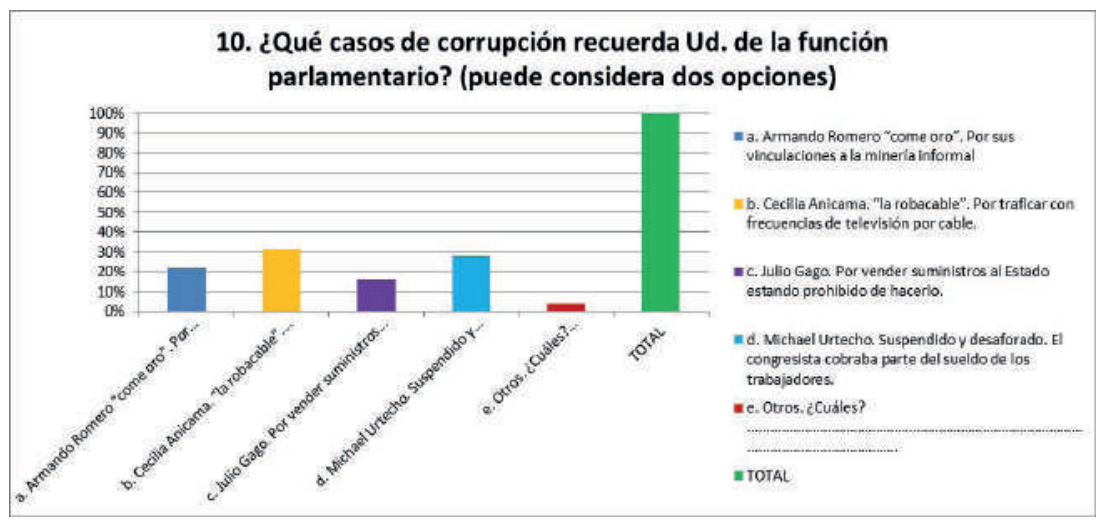

Respecto a algunos casos con la falta de ética de los parlamentarios, el (30.9\%) considera el caso de la congresista Cecilia Anicama, más conocida como la robacable, por retransmitir señales de cable no autorizados. Otro caso recordado por los estudiantes universitarios encuestados es el de Michael Urtecho (27.5\%), por la acusación contra él por parte de tres trabajadores, por haberse apropiado parte de sus sueldos. Un tercer caso recordado es el de Amado Romero, conocido como el comeoro (21.8\%). Al parlamentario se le vincula con la minería ilegal en Madre de Dios. De otro modo, el caso del congresista Julio Gagó, (15.9\%). Gagó encontró la manera de seguir licitando con el Estado, a pesar de que la ley de contrataciones prohíbe que un funcionario público como un congresista lo realice. Un (3.9\%) señala recordar otros casos. 


\section{Cuadro $\mathrm{N}^{\circ} 8$}

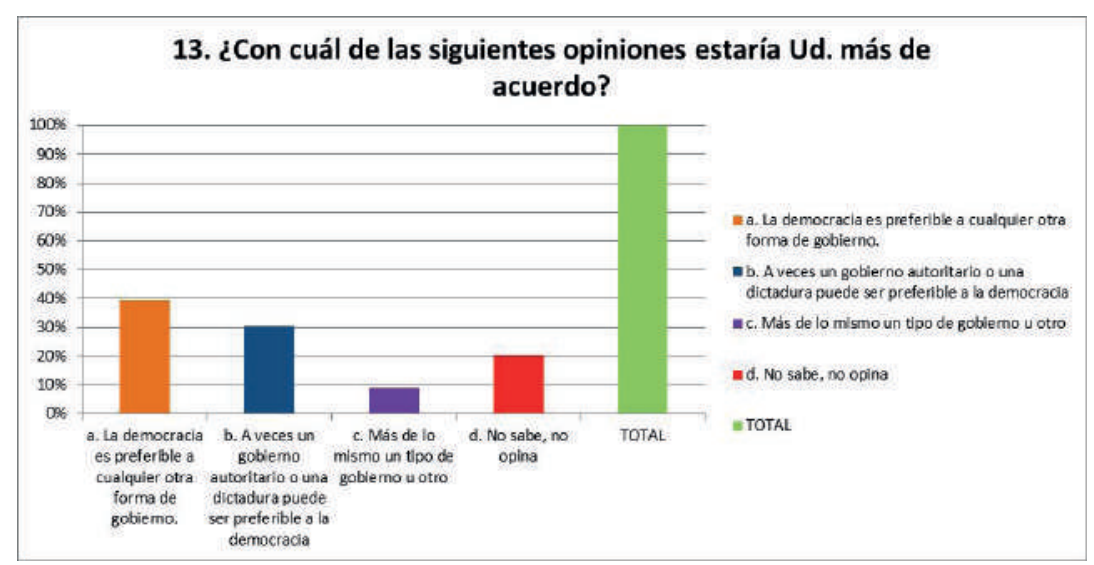

En el cuadro $\mathrm{N}^{\circ} 8$, se muestra la percepción de encuestados sobre los sistemas de gobierno, donde la democracia es el sistema de representación por excelencia de los estado-nación modernos (39.7\%). Un importante sector considera que es preferible a veces un gobierno autoritario o una dictadura (30.5\%). Mientras que un (8.8\%) de los encuestados, señala que las dos formas de gobierno son más de lo mismo, y un (20\%), no sabe no opina.

\section{DISCUSIÓN}

Las organizaciones políticas en el parlamento, no han canalizan los intereses y las perspectivas de los ciudadanos. Por otro lado, se observa una falta de interés y compromiso por parte de estos últimos hacia la rendición de cuentas. Esto puede ser explicado desde un menor o mayor nivel de información y consecuentemente en la participación de los hechos político que los conciernen.

Las nuevas organizaciones políticas carecen de una estructura orgánica en comparación a los partidos tradicionales. Los liderazgos no siempre están respaldados por un trabajo orgánico y canales de participación democrática. Por el contrario, se van dinamizando en periodos de campaña electoral. Se manifiesta un distanciamiento entre ciudadanos y parlamentarios respecto al proceso y elección, como mecanismos de participación. Otro elemento que puede explicar esta situación, es la proporción en la cantidad de congresistas que conforman la representación nacional, que actualmente es de cientotreinta parlamentarios. En países como Bolivia, Chile y Ecuador, la representación es mayor. Considerando entre otros aspectos; el criterio de la proporcionalidad electoral según circunscripciones y los mecanismos de elección de dos cámaras. De esta manera es importante la eliminación del voto preferencial y permitir un mecanismo de democracia y control interno dentro de las organizaciones, en la medida que permita elegir mejor a sus candidatos. 
Cuando el parlamentario es reelegido porque es conocido o mediático, se manifiestan algunos liderazgos, tanto individuales como colectivos dentro del grupo parlamento circunstancialmente pautados por la coyuntura política. Si son noveles la función legislativa, se desenvuelve en un inicial desconocimiento. En contraste con los primeros, los últimos, tienen la desventaja de no ser mediáticos o representantes conocidos, por poseer un menor capital político.

La imagen es un indicador eficaz de marketing político. El candidato al parlamento utiliza todos los recursos a su alcance, que le permiten mostrarse mejor que otros. Muchos son invitados a programas periodísticos, magazines y programas de la farándula. Otros son entrevistados en la radio. El uso de las redes sociales, se ha convertido en un medio eficaz de comunicación política el día de hoy. Donde a partir de las ideas y propuestas más ocurrentes se da el factor sorpresa, consiguiendo ser mediáticos ante la ciudadanía.

La corrupción en el Perú se entiende como un problema estructural, como histórico y cultural. Según los encuestados, tenemos en primer lugar la corrupción de funcionarios y autoridades, donde la desconfianza por parte del ciudadano en los agentes del Estado, se percibe como la posibilidad de servirse de la función. La eficacia de la gestión del Estado es indispensable en la medida que cuente con actores cualificados. Por el contrario, ." ...las mayorías absolutas minimizan el control de la oposición y crean la impresión de que los controles democráticos son débiles y acrecientan la posibilidad de impunidad..." (Calsamiglia 2015; 26). Pero la corrupción también es entendida como una venganza del funcionario frente al excesivo intervencionismo del Estado. Así, su actuación, como la ley, tiene un precio para el negociador público o privado, que establece algún tipo de sentido relacional. Por lo tanto, las instituciones del Estado permiten producir resultados emergentes pautados por acciones corruptas ya establecidas.

El parlamento peruano en los últimos treinta años, ha experimentado la transformación de nuevas formas de representación e institucionalización, desde pensar y actuar en el sistema político, como de su propia dinámica de funcionamiento por sus propios actores. Estos cambios se dan, respecto a su esquema institucional que teóricamente, tenía como base, la preminencia de ser órgano de representación ciudadana. Como parte del conjunto de creencias, valores y actitudes compartidos por lo miembros de una sociedad política, el rol de las actividades produce un cambio significativo, en los debates y las decisiones políticas que ya no constituyen su monopolio, y han aparecido nuevas instancias de representación y negociación de los intereses ciudadanos (Delgado, l; Martínez, A y P. Oñate 1998; 17). Están más ligados a la representación de la sociedad civil y su capacidad de negociación con la sociedad política.

Pero por otro lado, el escenario parlamentario hoy, es un espacio de escándalos, ligados a la falta de ética y de corrupción, y una de las razones que explica esta realidad, es la transformación de su cultura política, que paso de la promesa, al cinismo, de la disciplina partidaria a la preponderancia del individualismo y pragmatismos del espíritu de cuerpo partidario, a la no rendición de cuentas y la ausencia del poder fiscalizador, de la confrontación y entrampes, a la llamada 
eficacia dentro del papel subordinado y la desconexión con la base por parte de la cúpula (Degregori y Meléndez, 2007, 18)

Como parte de esta lógica de funcionamiento en el parlamento, se establece una nueva cultura política, que tiene en la corrupción, uno de los problemas centrales que afecta no solo el crecimiento, sino el desarrollo del país. No es la única institución afectada desde la percepción de los estudiantes universitarios. Lo es el Poder Ejecutivo, el Poder Judicial, la Policía Nacional y los partidos políticos, entre otras instituciones del Estado. Esta percepción está asociada al país también, desde la función de las autoridades y funcionarios, a la falta de interés de los ciudadanos, con el objeto de fiscalizar a los funcionarios y gobernantes, pero también la falta de recurso de los sectores más vulnerables economía y socialmente del país, y la falta de coordinación entre las distintas instituciones del Estado.

Si alguna persona procede de una determinada manera, es la sociedad desde su marco ético y moral, lo que determinará si su actuación fue correcta o incorrecta. La corrupción es, sin duda, la negación de la ética en la aspiración del justo medio. Constituye un conjunto de antivalores y costumbres que enajenan la acción positiva del ciudadano como: la deshonestidad, la injusticia, la discriminación, la falta de respeto a su dignidad, la hipocresía, la cobardía, la indecencia, el egoísmo, y la irresponsabilidad. Defectos que son inherentes, pero que degradan la persona y obstaculizan el desarrollo económico y social de un país.

De otro modo, los encuestados de las universidades, nos presentan sus apreciaciones sobre que comportamientos pueden ser considerando como faltos a la ética y de corrupción como; que un funcionario público favorezca a parientes y amigos, evadir impuesto si se sabe que no lo descubrirán, pagar propina para que le perdonen la multa, dar un obsequio o dinero para agilizar un trámite público. También es un acto corrupto, robar servicios públicos como el agua, la luz o el cable. A diferencia de entender la corrupción desde un sentido normativo y"oficial", desde una concepción amplia, los actores respetan determinadas reglas de juego, donde, por ejemplo, la reciprocidad no incluye regalos tangibles, pero si determinados favores entre relaciones de tipo horizontal, no condicionados ni exigidos. Esto se da esencialmente entre individuos que son de un mismo grupo social, lo cual permite encubrir la relación a partir de una "ideología de la amistad" o de compadrazgo, legitimando las relaciones sociales y humanas (Huber, 2008; 41).

En ese sentido, la Novena Encuesta Nacional para el 2015, elaborada para Proética por IPSOS Apoyo, como antecedente, confirma algunos datos de la investigación, donde:

“...el 46\% de los encuestado considera a la corrupción y las coimas como uno de los principales problemas del país. Es el problema más importante después de la delincuencia y la inseguridad ciudadana. Como problemas específicos del Estado, el $61 \%$ de los encuestados señala que fue la corrupción de funcionarios y autoridades. As mismo, cuatro de cada cinco entrevistados percibe que la corrupción se ha incrementado en 
los últimos cinco años. Así mismo, el 53\% espera que este aumente en el quinquenio siguiente" (Proetica, 2015).

Los casos emblemáticos que permiten comprender el tema de la corrupción, esta mediado por prácticas culturales que son construidas entre el elector y el representante y mediatizados por los medios de comunicación y las redes sociales. Al margen de una apreciación normativa y económica de la corrupción, son las relaciones sociales y el funcionamiento de las instituciones, lo que permite legitimar los actos corruptos (Rose-Ackerman 2001). Así los encuestado señalan como casos significativos de escándalos, faltos a la ética y de corrupción del parlamento peruano 2011-2016; el caso de la congresista Cecilia Anicama, por traficar con frecuencias de televisión por cable en la Provincia de Pisco. El caso de Amado Romero, por sus vinculaciones con la minería ilegal en Madre de Dios. Michael Urtecho, por cobrar parte del sueldo a sus trabajadores del despacho parlamentario (Torres 2014) y Julio Gagó, por licitar con el Estado, cuando esta prohibido como funcionario público, de hacerlo.

El ciudadano no confía en sus intuiciones, siendo proclives a pensar que el parlamento no los representa políticamente. Los escándalos mediáticos, que linda con la ética y el delito, de personajes públicos que transgreden las normas sientan las bases para optar por opciones autoritarias. Así, algunos de los escándalos vistos, no se dan como parte de la función pública, sino que se dieron con anterioridad a ella. La preocupación incide en la legitimidad del parlamento como parte del sistema democrático. También los encuestados entienden otras vías no democráticas por incrementar su participación política.

La historia del Perú ha demostrado que hay más evidencias de corrupción. Desde el virreynato como en la república, en gobiernos civiles como militares (Quiroz 2013). El país no refleja por tradición una cultura política democrática. Lo que lo hace más previsible a la política de caudillos, outsider, gobernantes autoritarios e improvisados por el pragmatismo electoral y las limitaciones del propio sistema. El mismo, evidencia procesos de interrupción de las instituciones democráticas, lo que no lo hace insoslayable a la corrupción, bajo la idea de la mano fuerte, o el supuesto orden y la aparente fiscalización de las instituciones del Estado desde el poder.

\section{BIBLIOGRAFÍA}

Bernales, E. (2002). Parlamento y ciudadanía. Problemas y alternativas. Lima: Comisión Andina de Juristas.

Bernales, E. (1990). Parlamento y democracia. Lima: Editorial Hipatia S. A.

Calsamiglia, A. (2015). Cuestiones de lealtad: límites del liberalismo: corrupción, nacionalismo y multiculturalismo. Barcelona: Paidós Ibérica.

Campos, M. (2017)."Los grupos parlamentarios como instituciones de intermediación entre partidos políticos y Congreso". Elecciones, 16(17), 139-167. 
Casado, M. (2018). “Representación política: El ciudadano como protagonista del cambio". En: Vox Juris, 35(1), 137-143.

Congreso de la Republica. (2020). Reglamento General del Congreso de la Republica. Lima: Edición Oficial.

Congreso de la Republica. (1993). Constitución Política del Perú. Lima: Diario Oficial El Peruano.

Delgado-Guembes. (2012). Manual del Parlamento: introducción al estudio del Congreso peruano. Lima: Congreso de la Republica del Perú, Oficialía Mayor.

Delgado, I.; Martínez, A. y P. Oñate. (1998). Parlamento y opinión publica en España. Madrid: Centro de Investigaciones Sociológicas.

Degregori, C. y C. Meléndez. (2007). El nacimiento de los otorongos. El Congreso de la República durante los gobiernos de Alberto Fujimori (1990-2000). Lima: Instituto de Estudios Peruanos.

Durkheim, É. (1988): Las reglas del método sociológico y otros escritos sobre filosofía de las Ciencias Sociales. Madrid: Alianza Editorial.

Guimaray, E., \& Prado, B. (2020). Corrupción Política parlamentaria en el Perú. En Desafíos actuales del derecho: aportaciones presentadas al II Congreso Nacional de jóvenes investigadores en Ciencias Jurídicas (pp. 303-319). EUMED.

Huber, L. (2017). Corrupción y transparencia. Balance de Investigación en Políticas Públicas 2011-2016 y Agenda de Investigación 2017-2021, 2017, 20.

Huber, L. (2008). Romper la mano. Una interpretación cultural de la corrupción. Lima: Insituto de Estudios Peruanos.

IDEA/UARM. (2009). Estudios sobre el Congreso Peruano. Grupos parlamentarios, disciplina partidaria y desempeño profesional. Lima: IDEA/UARM.

Jurado Nacional de Elecciones. (2011). Elecciones Generales 2011. Estadísticas, resultados. Lima: Direcciones de Registro, Estadística y Desarrollo Tecnológico.

Klitgaard, R. (1990). Controlando la corrupción. La Paz: Editorial Quipus.

Meléndez, C. (comp) (2011). Anti-candidatos: guía analítica para unas elecciones sin partidos. Lima: Aerolíneas Editoriales.

Morelos, J. (2015). La corrupción. Conceptos, causas, consecuencias, tipos y áreas críticas. Disponible en: <http://www.imagenpolitica.com/men6.html l>

Mujica, J. \& Zevallos, N. (2016). Reducir la pequeña corrupción en los servicios de atención a la ciudadanía. Elementos conceptuales y marco lógico para el diseño de una intervención. En: Serie Cuadernos de investigación, № 1. Lima: Escuela de Gobierno y Políticas Públicas de la Pontificia Universidad Católica 
del Perú, en línea < http://escuela.pucp.edu.pe/gobierno/publicaciones/ reducir-pequena-corrupcion $>$ [consultado el 17 de agosto de 2020].

Mujica, J. (2013). Representatividad, representabilidad, el problema de la representación política. Hacia una etnografía del parlamento peruano. s/f, en línea:< http://escuela. pucp. edu. pe/gobierno/images/.../articulo2_jaris_ mujica. doc> [consultado el 17 de agosto de 2020].

Proética. (2015). Novena Encuesta Nacional sobre Anticorrupción. Disponible en: https://www.proetica.org.pe/noticias/novena-encuesta-nacional-sobrepercepciones-de-la-corrupcion-2015/ [consultado el 18 de agosto de 2020].

Quiroz, A. (2013). Historia de la corrupción en el Perú. Lima: IEP. Instituto de Defensa Legal.

Rose-Ackerman. R. (2001). La corrupción y los gobiernos. Causas, consecuencias y reforma. Madrid: Siglo XXI de España editores, S. A.

Torres, D. (2014). "el caso Michel Urtecho". En: Crónica Judicial, Proyecto Anticorrupción del IDEHPUCP, PUCP.

Ugarteche, O. (compilador) (2006). Vicios públicos. Poder y corrupción. Lima: Fondo de Cultura Económica/ Casa Sur.

Valencia, I. (2007). La representación parlamentaria. Un recorrido histórico por la teoría política. México: Universidad Autónoma Metropolitana.

Yalle, O. (2019). Representación Parlamentaria y corrupción en el Perú. El caso de los Parlamentarios 2011-2016. En: Scientia, 21(21), 99-116. https://doi. org/10.31381/scientia.v21i21.2762

Zegarra, L. (2002). La economía de la corrupción: hacia una comprensión de las causas de la corrupción y las estrategias ora combatirla. Lima: Universidad del Pacífico. 